\title{
Comparative evaluation of different cell disruption methods for the release of recombinant hepatitis B core antigen from Escherichia coli
}

\begin{abstract}
A comparative evaluation of five different cell-disruption methods for the release of recombinant hepatitis B core antigen $(\mathrm{HBcAg})$ from Escherichia coli was investigated. The cell disruption techniques evaluated in this study were high-pressure homogenization, batchmode bead milling, continuous-recycling bead milling, ultrasonication, and enzymatic lysis. Continuous-recycling bead milling was found to be the most effective method in terms of operating cost and time. However, the highest degree of cell disruption and amounts of $\mathrm{HBcAg}$ were obtained from the high-pressure homogenization process. The direct purification of $\mathrm{HBcAg}$ from the unclarified cell disruptate derived from high-pressure homogenization and bead milling techniques, using batch anion-exchange adsorption methods, showed that the conditions of cell disruption have a substantial effect on subsequent protein recovery steps.
\end{abstract}

Keyword: Batchanion-exchange adsorption; Bead milling; Hepatitis B core antigen; Highpressure homogenization; Lysozyme; Ultrasonication 